\title{
THE TREATMENT OF PULMONARY TUBERCULOSIS IN ADULTS BY RESECTION
}

\author{
BY \\ H. T. THOMPSON, T. SAVAGE, AND T. H. L. ROSSER \\ From Sully Hospital, Glamorgan
}

(RECEIVED FOR PUBLICATION OCTOBER 23, 1953)

It has been shown by Overholt, Wilson, and Gehrig (1952) and others in America, and Bickford, Edwards, Esplen, Gifford, Mair, and Thomas (1951) and others in this country, that pulmonary resection has a place in the treatment of pulmonary tuberculosis.

A series of 266 resections of various kinds, performed on 259 patients at Sully Hospital, is presented. It is intended to show that resection is now a relatively safe procedure, and that the early results have been satisfactory.

\section{INDICATIONS FOR RESECTION}

As time has passed the indications for resection have broadened. Taking into account the pathological state of the lung alone, in our opinion, resection is indicated in the following types of lesion: (1) The tuberculous cavity, (2) the solid tuberculous lesion, (3) tuberculous bronchiectasis, and (4) tuberculous bronchostenosis. Any of these lesions may be associated with a tuberculous empyema or may have been treated unsuccessfully with some form of collapse therapy, e.g., a thoracoplasty or artificial pneumothorax.

UNILATERAL RESECTION.-In cases of unilateral disease, pneumonectomy would be performed where any of the above pathological processes involve the whole lung and lobectomy where they involve a whole lobe. Segmental resection would be used where one or two adjacent segments are involved, or occasionally where two widely separated segments only are involved, or in association with a lobectomy, the commonest combination being the apical lower lobe segment with an upper lobectomy. Occasionally segmental resection is performed in the presence of widespread disease where it is thought that the segment involved acts as a focus of infection. This applies especially to lesions in the apical segment of the lower lobe which are not amenable to other forms of treatment. Wedge resection is now used only for multiple small solid lesions or for a single solid lesion in the remaining lobe after segmental resection or lobectomy. Before segmental resection became a practical procedure, local excision of a cavity (spieliectomy) or local suture of a cavity (spieliorrhaphy) were performed.

Bilateral Resection.-The indications we have used are as for unilateral resection. If bilateral surgery has been planned the lesser resection is performed first wherever possible, so as to obtain a relatively sound lung on the opposite side before embarking on the major resection. The following combinations are considered as practical possibilities: (1) Resection of one segment of one lung before pneumonectomy is performed on the other side. (2) One or two segments of one lung may be resected with resection of a lobe of the opposite lung. (3) Bilateral segmental resection may be performed. (4) Bilateral lobectomy may be performed.

\section{Pre-operative InVestigation}

SPutum Examination.-All cases are subjected to a rigid sputum examination, 14 specimens of morning sputum being subjected to direct examination and culture in the first instance. If there is no sputum the same investigation is performed on the gastric lavage. If these prove to be negative, further sputum or gastric lavage examinations are carried out where the indication for resection is doubtful.

On all positive cases streptomycin sensitivity tests are performed, and where there has been any previous treatment with antibiotics, sensitivity of the organism to I.N.A.H. and P.A.S. is determined. Streptomycin resistance is not considered an absolute contraindication to resection, but if there is another method of treatment available, this is to be preferred.

RADIOGRAPHY.-Postero-anterior and lateral radiographs are taken routinely as well as postero-anterior tomographs in most cases. 
Lateral tomography is now used more widely, particularly in those cases where segmental resection is contemplated, as this method localizes the lesions more accurately. Bronchography is performed on all cases for segmental resection or lobectomy, partly for information on the pathological state of the bronchi and partly for establishing the segmental anatomy of the part to be resected.

BRONCHOSCOPY.-This is not performed as a routine in all cases, but is confined to those where it is thought that there may be active tuberculous endobronchitis, or bronchostenosis affecting a major bronchus.

Special Respiratory Function Tests.-These are performed only on cases of doubtful respiratory efficiency. The maximum breathing capacity is the test we perform in this type of case. When particular knowledge of the relative function of each lung is required, bronchospirometric measurements are taken of the ventilatory capacity and relative oxygen uptake of each lung. All these tests are taken into account in the general clinical assessment.

\section{Pre-operative Preparation}

Apart from the general measures taken in the preparation of any patient for major surgical treatment, the following measures need special consideration.

Postural Treatment.--This (Dillwyn Thomas) method is used only in cases of cavitated disease. The aims are: (1) Closure of the cavity so that there is less danger of a spill-over spread at the time of the operation; (2) the prevention of spread to other parts of the lungs during the time of waiting for surgical treatment; (3) as a test for revealing a doubtful cavity on the "good" side, particularly where pneumonectomy is contemplated on the "bad" side. After a short period on postural treatment in which the doubtful lung is uppermost a cavity usually becomes demonstrable if it exists. If this possibility is anticipated, radiographs should be taken after one month, and if cavitation becomes obvious the particular posture in use should be immediately abandoned.

In using postural treatment the cavity is accurately localized to its segment and the appropriate posture instituted for its treatment. The various positions which are maintained to close or reduce cavities are as follows:

For cavities in the apex of the lower lobe or in the posterior segment of the upper lobe, the patient lies with the cavity dependent and with the body inclined backwards at an angle of $35^{\circ}$ to the horizontal, and with the foot of the bed raised so that the feet are above the level of the head as in Fig. 1.

Cavities in the apical segment of the upper lobe and laterally placed cavities are treated with the patient lying on the affected side at $90^{\circ}$ to the horizontal and with the feet raised so that the cavity is dependent as in Fig. 2.

Anterior cavities are treated with the patient lying on the face at $35^{\circ}$ to the horizontal with the cavity dependent and with the foot of the bed raised as in Fig. 3.

Bilateral apical cavitation is treated by lying the patient on his back with the foot of the bed raised.

Postural treatment is instituted as soon as the patient is admitted to hospital and is maintained until the cavity appears to be closed and then the resection is performed.

CHEMOTHERAPY.-Together with postural treatment, chemotherapy is used to produce maximum stability, and, wherever possible, resection is not performed until the lesion no longer shows any sign of improvement. Most patients coming to resection have been treated with some form of chemotherapy and no further course is given unless there are signs of activity, e.g., tuberculous endobronchitis. In all other patients chemotherapy is withheld until the time of operation when, seven to 10 days before resection, $1 \mathrm{~g}$. of streptomycin with 20 g. of P.A.S. are given daily. This routine is carried on through the operation for a total of 21 days and then streptomycin, $1 \mathrm{~g}$. twice weekly, and P.A.S. daily are given for a further nine weeks. This treatment may vary slightly in different circumstances but is the routine in the straightforward case. Penicillin is also used as a cover in all patients.

At the present time the combination of streptomycin and P.A.S. appears to have been adequate, and only in cases of streptomycin resistance or intolerance has any other combination been used.

Physiotherapy.-Before operation the patient is given instruction in lower costal and diaphragmatic breathing exercises and coughing.

\section{Operative Technique}

Position of the Patient on the Table.-The lateral position is used as a routine for resections. Many workers, notably Overholt, Langer, Szypulski, and Wilson (1946), Bailey (1947), and Holmes Sellors and Hickey (1949), have advocated the face-down position, but in our hands the incidence of operative spreads appears to be no higher using the lateral position. 


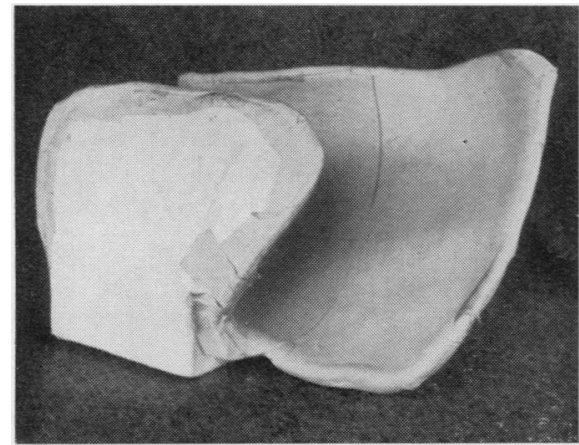

FIG. $1 a$

FIG. 1a.-Plaster cast with $35^{\circ}$ backward tilt for reduction of cavities in the apical segment of lower lobe or posterior segment of upper lobe of left lung. (b) Patient in the left posterior posture.

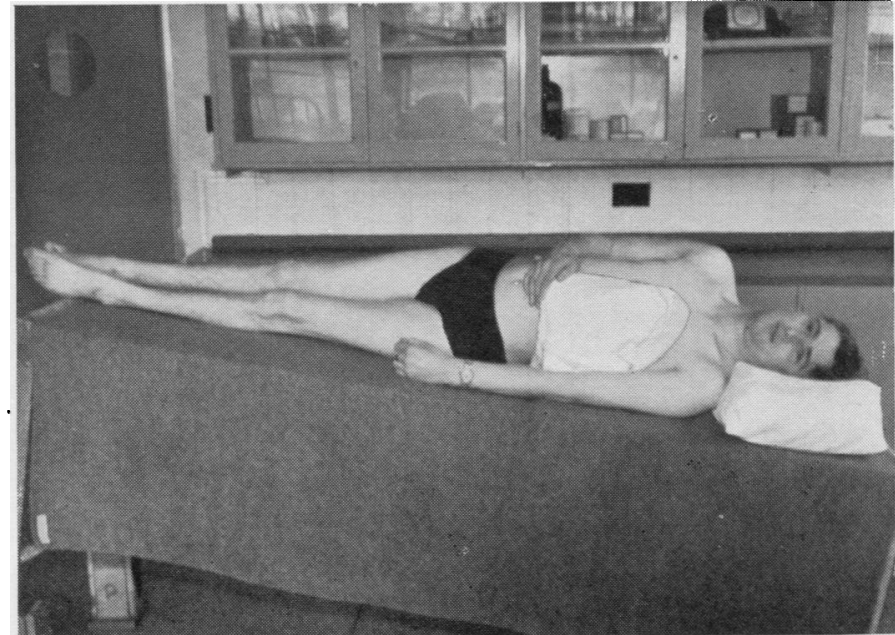

Fig. $1 b$

ANAESTHESIA.--Nitrous oxide and oxygen supplemented with intravenous pethidine and flaxedil has been the anaesthetic used. For the past two and a half years pulmoflators of the "blease" type have been in use. In our opinion, these mechanical respirators, while ensuring adequate oxygenation of the patient, produce ideal working conditions for the surgeon.

When the operation is completed any bronchial secretions are aspirated and the lung fully reexpanded. Post-operative bronchoscopy is not routinely carried out.

The Operation.-A standard postero-lateral thoracotomy approach is used; the first step on entering the pleural cavity is to free any peripheral attachments of the lung and in particular the pulmonary ligament. Objections to this technique may be raised on the grounds of increased risk of operative spread. This has not been found to be so, and we believe that it makes for safer operating if the hilum is easily controlled when the pulmonary artery and its branches are being dissected. The technique of closing the bronchus is similar to that described by Overholt and others (1946) and Bailey (1947).

The following points, which apply especially to pneumonectomy, are also used where possible in other resections. (1) In clearing the bronchus care
FIG. 2a.-Plaster cast for the reduction of laterally placed cavities and also those in the apical segment in the upper lobe of the left lung. (b) Patient in the left lateral posture.

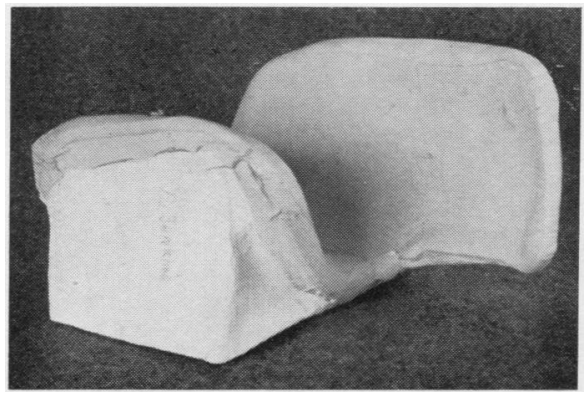

Fia. $2 a$

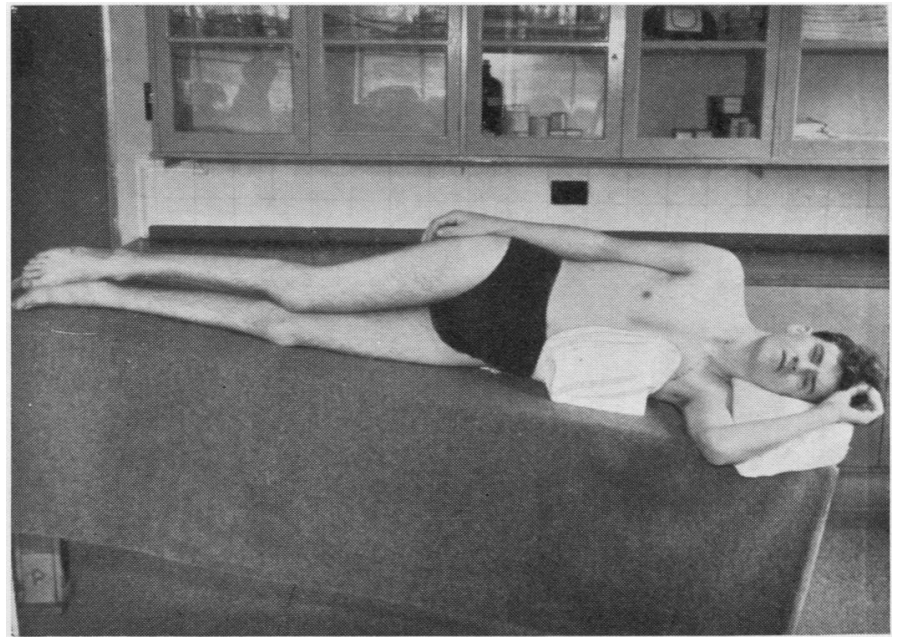

FIG. $2 b$ 


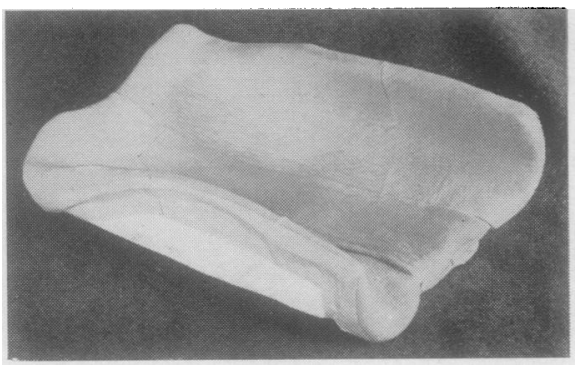

FIG. $3 a$

Fig. 3a-Plaster cast with a $35^{\circ}$ forward tilt for the reduction of anteriorly placed cavities in the left lung. (b) Patient in the left anterior 'posture.

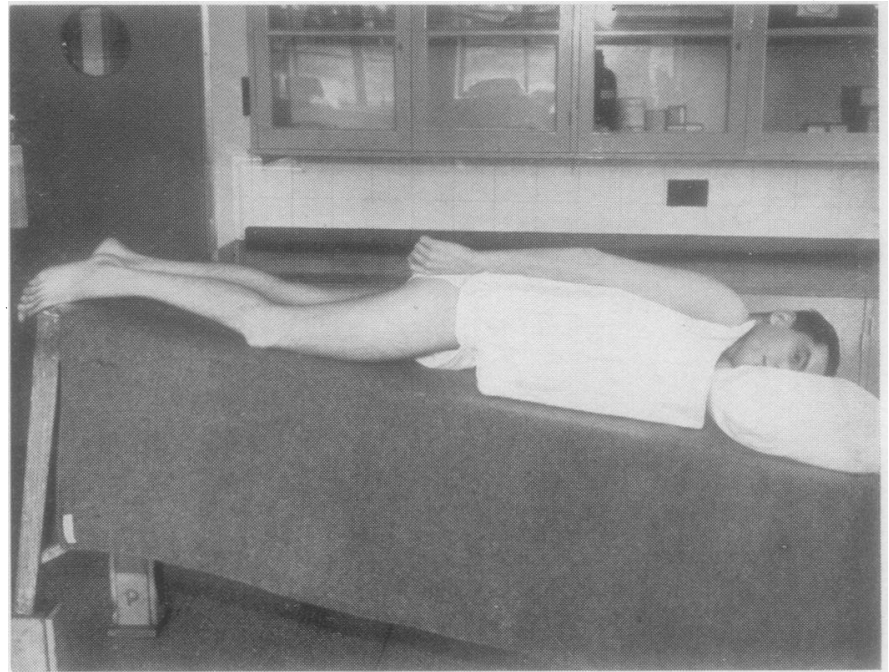

FIG. $3 b$

After trials with many other varieties of tubing this type has been found to give the most satisfactory results. After pneumonectomy the pleural space is drained by a basal tube placed in the posterior axillary line and connected to an underwater seal. In using this procedure haemorrhage is not concealed and the patient is safeguarded should an early broncho-pleural fistula occur. There is no danger of a tension pneumothorax as a result of leakage of air from the suture holes in the bronchial stump and there is no necessity for post-operative aspiration.

For all other resections two tubes are used. The basal tube is placed in the same way as the
FIG. 4a.-Plaster cast for reduction of bilateral cavities.

(b) Patient in the posterior plaster cast.

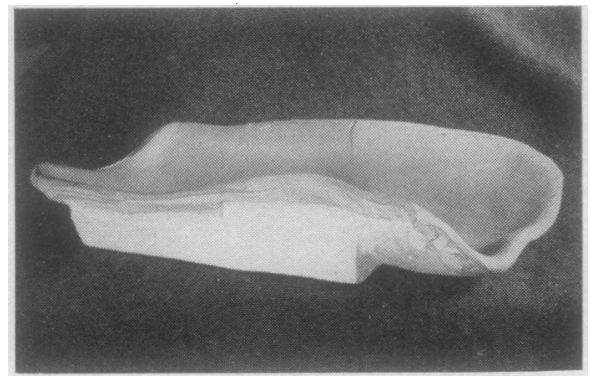

FIG. $4 a$

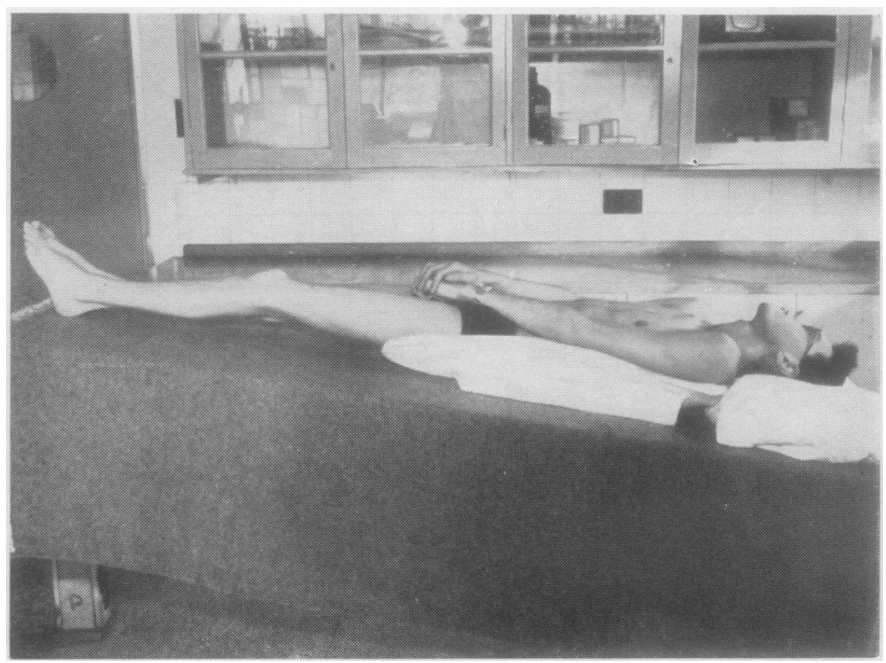

Fig. $4 b$ 
tube for drainage following pneumonectomy, but inside the pleural cavity it is kept against the chest wall by a sling of catgut, passed through the appropriate intercostal space, to prevent occlusion of the tube by lung or diaphragm. The apical tube is placed in the same interspace as the basal tube, in the mid-axillary line, and runs up inside the chest wall to the apex. It is kept against the chest wall by two catgut sling sutures passed through intercostal spaces above and below the thoracotomy incision. Both tubes are anchored to the chest wall by the device shown in Fig. 5 . This plaque of perspex is sutured to the skin, and,

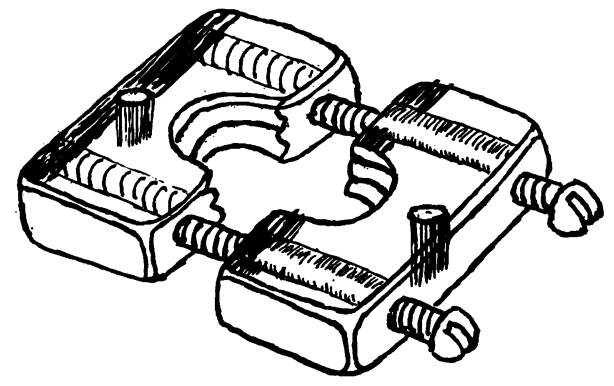

Fig. 5.-Perspex tube holder.

when screwed up tightly, grips the tube firmly without occluding the lumen. This holder saves the tube being transfixed by a suture and also enables the tube to be shortened without difficulty in the post-operative period.

Post-Resection Thoracoplasty. - Thoracoplasty is carried out after pneumonectomy for the following reasons. Space obliteration is considered to be of paramount importance in the prevention of late broncho-pleural fistula. Even without a broncho-pleural fistula an empyema may form in a pneumonectomy space. Thoracoplasty is not only prophylactic, but should an empyema have already developed, it will also act as a therapeutic measure. Thoracopasty prevents mediastinal displacement and overdistension of the remaining lung.

An eight-rib thoracoplasty is performed in one stage, two weeks after pneumonectomy. The eighth rib is removed to make certain the scapula is embedded. In some cases the first rib has been left. This makes for a straighter neck, but increases the risk of a residual space. In all cases the pleural space is opened and blood clot evacuated. A large musculo-periosteal flap is then made, hinging posteriorly, and this is allowed to fall back into the paravertebral gutter to cover the hilum. To make sure that this flap stays against the mediastinum, the space is drained for
48 hours by a basal tube connected to a Robert's pump.

Apical corrective thoracoplasties are performed after lobectomy, or following multiple segmental resections where a large volume of lung has been removed, where it has been necessary to leave palpable disease in the remaining lung tissue, and where there is a persistent apical space following resection.

The thoracoplasty, consisting of three, four, or five ribs depending on individual indications, is usually performed three to six weeks after the resection. It is preferred to phrenic crush on the grounds that the reduction in chest volume is permanent, but is less function-destroying (Himmelstein, Berry and Read, 1950). Following lower lobectomy a phrenic crush is still the usual procedure where the space is large.

MANAGEMENT OF THE STREPTOMYCIN-RESISTANT CASE.-The two important complications that are feared in the streptomycin-resistant case are operative spread to the contralateral lung and development of a broncho-pleural fistula.

Where pneumonectomy is contemplated the first step is the performance of a four-rib thoracoplasty with the patient sitting up (lateral position with the table tilted to more than $45^{\circ}$ so that any spread will be ipsilateral). Two weeks later the pneumonectomy is performed and the thoracoplasty completed and a muscle flap sutured over the bronchus.

In the case of other resections, generally speaking, streptomycin resistance is an indication for other forms of therapy, but where this is found to be unsuccessful, resections have been carried out, reliance being placed on rapid and complete reexpansion of the remaining pulmonary tissue.

\section{Post-operative Care}

It is considered that the essential aim of treatment in the post-operative care following pulmonary resection is obliteration of the residual pleural space, and in segmental or lobar resection this implies rapid re-expansion of the remainder of the lung. In order to ensure this, all patients are nursed in a special post-operative ward, staffed by nurses with a knowledge of the method used. The following measures are important in achieving these aims.

Management of Intercostal Drainage.After lobectomy and segmental resection continuous suction of approximately half an atmosphere pressure is applied through a water seal to both drainage tubes. By this means all the fluid 


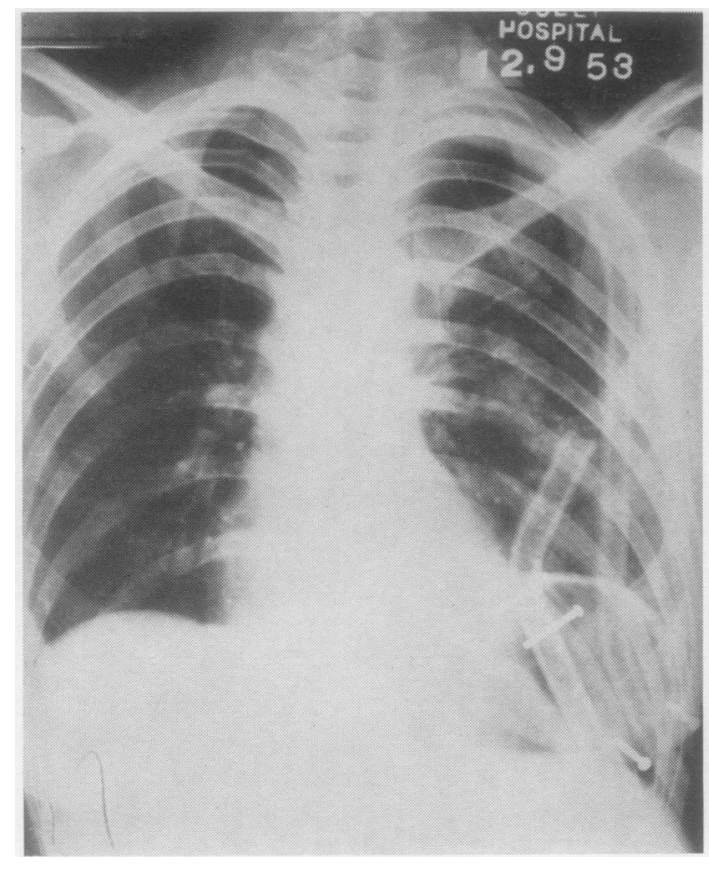

FIG. $6 a$

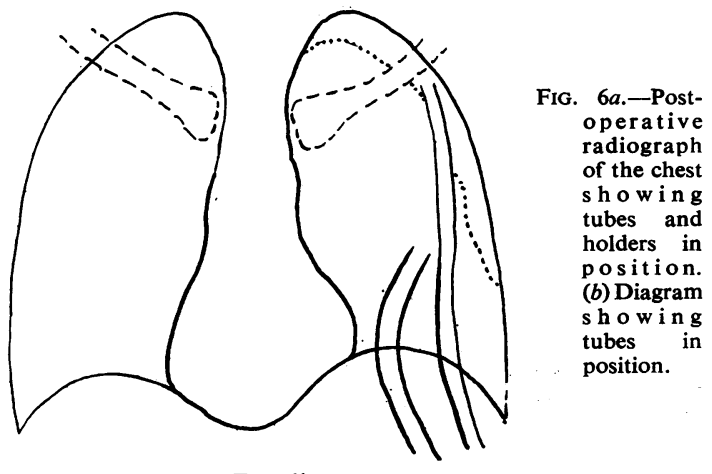

FIG. $6 b$

collecting in the chest is evacuated, and the lung is enabled to expand in the face of any leak from it. By remaining in constant close approximation with the chest wall, fluid is prevented from accumulating, and "alveolar" leakage of air is stopped. The tubes are removed when the space is completely obliterated, the lung is fully expanded, and air has finally stopped leaking from the lung. Following lobectomy this is usually achieved after 48 to 72 hours and following segmental resection after three to five days, or in the occasional case even longer. Until this has occurred, continuous suction is maintained through the tubes (Fig. 6).
After pneumonectomy the one basal tube is attached to an under-water seal without suction and left in for 48 hours in the straightforward case.

Position of the Patient.-After lobectomy and segmental resection the patient is nursed lying on the sound side over a pillow. This places the operated lung in a position of inflation and facilitates bronchial drainage. This position, in our opinion, in association with continuous suction, ensures rapid and complete re-expansion.

After pneumonectomy the patient is nursed with the sound side uppermost.

Coughing.- This is the main factor in the prevention of atelectasis and is encouraged as soon as the patient recovers consciousness; thereafter coughing is supervised at hourly intervals for the first 48 hours.

Post-OPerative Radiographs.-A portable antero-posterior radiograph of the chest, with the patient sitting up in bed, is taken from four to six hours after operation, then at 12-hourly intervals until the tubes are removed, and thereafter as required. This routine ensures the early detection of atelectasis, or the appearance of a pleural space.

\section{Complications and Their Management}

HAEMORRHAGE.-Normally, drainage of the chest by the method described deals adequately with any blood in the pleural cavity. Occasionally, where loss of blood has been excessive and clotting has occurred, it has been necessary to open the chest, evacuate the clot, and deal with the bleeding point. Rarely has it been necessary to aspirate the chest following the method of drainage now in use.

SuRgical EMPhySEMa.-With the method of drainage used, this complication is rare and can be treated by adjustment of the tubes, or if necessary by inserting a new intercostal tube into the pleural cavity and applying adequate suction.

Atelectasis.- The main factor in the prevention of this complication is adequate productive coughing. If atelectasis occurs, the following routine is carried out immediately. Vigorous productive coughing with postural drainage under supervision is demanded of the patient. Should the patient be unable to respond and subsequent radiography show that the atelectasis persists, the mucous membrane of the trachea is stimulated by the passage of a gum elastic catheter through the cords by direct laryngoscopy as shown in Fig. 7. In our opinion this method is superior in its therapeutic effect to suction by bronchoscopy. 


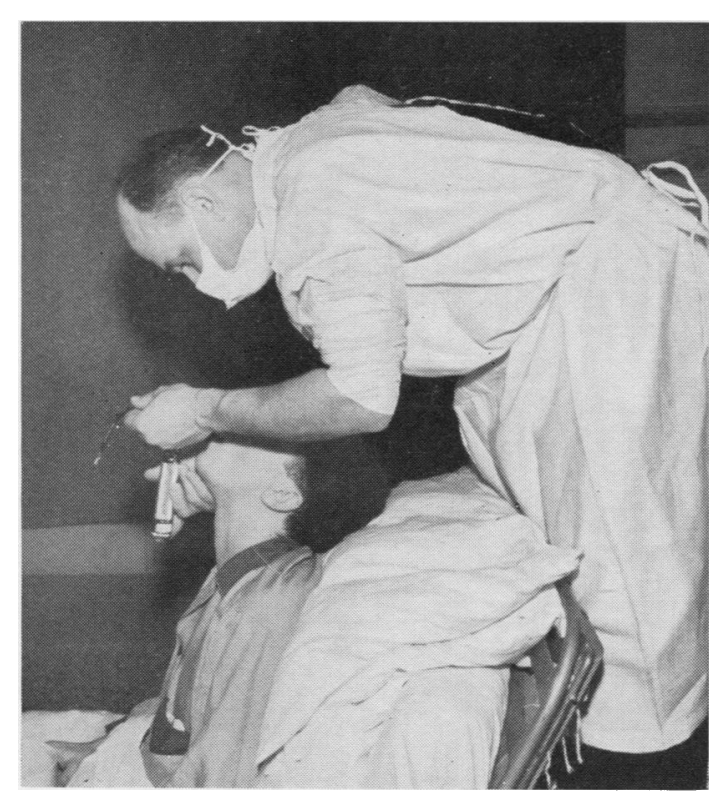

FIG. 7.-Position of the patient and operator for tracheal intubation

SPREAd of Disease.-Pre-operative measures are designed to prevent this. If a spread does occur, pre-operative sensitivity tests of the organism are of value in treating this complication with the appropriate antibiotic. It is in those cases where the organism has been resistant to streptomycin that the consequences have been serious. If the organism is sensitive to streptomycin the standard routine is modified and the drug given as dictated by the progress of the lesion.

Broncho-Pleural Fistula.- - It is believed that the most effective prevention of this complication is adequate bronchial closure as described and quick obliteration of the pleural space. If this complication occurs as shown by coughing of blood, fever, and the appearance of a pleural space about the tenth day, the chest is opened immediately and the fistula repaired, all necessary measures being taken for rapid re-expansion of the lung in the case of segmental resection and lobectomy. In cases of fistula following pneumonectomy the same procedure of repair is followed and the space is obliterated by corrective lateral thoracoplasty with a musculo-periosteal flap similar to that previously described.

"Alveolar" Leaks.-These are differentiated from broncho-pleural fistula in that there is no coughing of blood and no fever in association with the appearance of a pleural space. Many are treated by removing air with the artificial pneumothorax apparatus ; if this treatment is not immediately successful an intercostal tube is inserted and the air removed by continuous suction. Apical spaces which are persistent are treated with a small three- or four-rib thoracoplasty three to four weeks after the original resection.

EMPYEMA.-Total excision of the empyema cavity is the treatment of choice for empyema following lobectomy and segmental resection. In the past other methods have been used, but excision has been found to be superior in its results.

After pneumonectomy drainage is instituted, and when the patient is fit enough the space is obliterated by the usual corrective thoracoplasty and flap.

\section{Review of Sully Cases}

Two hundred and sixty-six resections have been performed on 259 patients over 15 years of age. There were five early post-operative deaths (within three months) giving an operative mortality of $1.88 \%$ and two later deaths giving a total mortality to date of $2.63 \%$. One other death was from a cause unrelated to chest disease. The period of follow-up is from three months to five years. At present 247 patients $(94.97 \%)$ are well and sputumnegative and have no signs of active disease. One hundred and sixty are working, 68 are at home but not yet working, and 19 are awaiting discharge from hospital.

TYPE OF RESECTION.-Table I sets out the number and types of resections performed at Sully over five years until June, 1953. It will be seen that there has been a steady increase in the more conservative type of resection, and fewer pneumonectomies are performed. There are two reasons for this. One is that fewer destroyed lungs are seen, and second that with the improvements in

TABLE I

NUMBER AND TYPE OF RESECTIONS IN FIVE YEARS ENDING JUNE, 1953

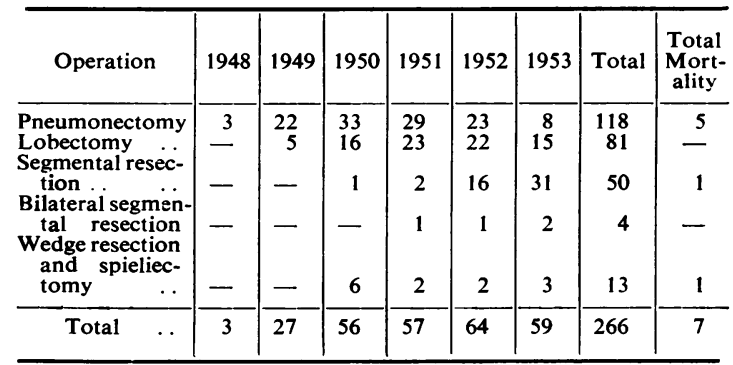


operative technique and post-operative care more conservative resections can be carried out.

MorTality.-Table II sets out the mortality both early (up to three months post-operatively) and late for all resections. Apart from those cases shown in the table, there has been one other death from causes other than pulmonary tuberculosis.

TABLE II

MORTALITY IN 266 RESECTIONS

\begin{tabular}{|c|c|c|c|}
\hline Operation & Early & Late & Total \\
\hline $\begin{array}{lcc}\begin{array}{l}\text { Pneumonectomy } \\
\text { Lobectomy }\end{array} & \ldots & . \\
\text { Segmental resection } & \ldots & \ldots \\
\text { Wedge resection and spieliectomy }\end{array}$ & $\frac{3}{1}$ & $\underline{2}$ & $\frac{5}{1}$ \\
\hline
\end{tabular}

Post-pneumonectomy Deaths.-The causes of post-pneumonectomy deaths were as follows: Pulmonary oedema ; pulmonary oedema following ligature of a false aneurysm of the first part of the subclavian artery injured during the corrective thoracoplasty, possibly due to overloading of the circulation; pulmonary embolism following corrective thoracoplasty; broncho-pleural fistula with contralateral spread of disease in a patient with a streptomycin-resistant infection ; bronchopleural fistula developing four months after pneumonectomy, corrective thoracoplasty having been considered inadvisable because of the patient's poor general condition. When the fistula developed, a corrective thoracoplasty and flap operation was performed, but the fistula persisted and eventually involved the oesophagus. The patient died of amyloid disease some two years later.

Deaths after Other Resections.-Two patients of this category died. The first was a case of a fulminating gangrenous staphylococcal infection. This patient was a young man who had the apicoposterior segment of the left upper lobe resected. The post-operative radiograph showed an opacity in the operation area which was thought to be a haematoma. On the third post-operative day his temperature rose steadily, and, in spite of aureomycin therapy, on the sixth post-operative day it reached $104^{\circ} \mathrm{F}$., when he showed signs of severe toxaemia with delirium. During this period the radiological opacity had not increased in size. The chest was reopened with a view to draining what was thought to be an infected haematoma. At operation there was a small amount of free fluid, the anterior segment of the upper lobe was found to be gangrenous, and the veins draining this segment were thrombosed. The remainder of the upper lobe was resected, but the patient did not recover. The necropsy showed a marked tracheobronchitis and a penicillin-resistant staphylococcus was isolated.

The second was a case of massive haemorrhage following a wedge resection. The patient had a haemorrhagic diathesis and died in spite of blood transfusion.

MorBiDITY.-The important complications following resection and peculiar to this form of surgery are set out in Table III.

TABLE III

COMPLICATIONS IN 266 RESECTIONS FOR PULMONARY TUBERCULOSIS

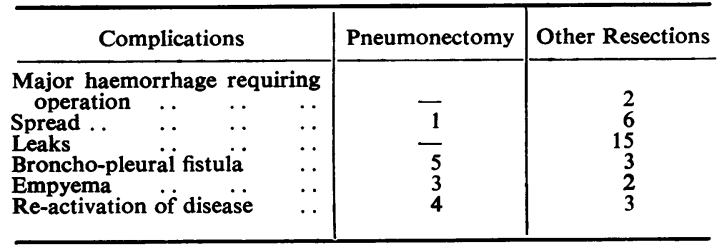

HAEMORRHAGE.-There have been two cases where haemorrhage has necessitated thoracotomy for evacuation of blood clot. One of these cases has already been mentioned in the section on mortality.

SPREADS.-There have been seven cases of operative spread in the series, six of which followed lobectomy and one pneumonectomy. All cases have cleared radiologically after streptomycin and P.A.S. All are now well and sputum negative.

LEAKS.-Fifteen cases have had persistent "alveolar" leaks which have sealed off within a few days on suction. In some cases persistent apical spaces have been left, these being closed later by an apical corrective thoracoplasty.

BRONCHO-PLEURAL Fistula.-There have been five post-pneumonectomy fistulae and three following other resections. Apart from the two pneumonectomy cases mentioned in the section on mortality, the others have responded to treatment and are now fit and well.

EMPYEMA.-Three pneumonectomy cases developed pyogenic empyemata without fistula formation. These all occurred in the immediate postpneumonectomy period and were successfully treated by drainage and corrective thoracoplasty. Following other resections two cases of empyema were successfully treated by excision.

Re-activation of Disease.-Of four patients who had pneumonectomies performed in the 
presence of contralateral disease, in three the disease is active and in one is quiescent.

Following other resections three patients had signs of disease elsewhere. Two of these have subsequently had residual pneumonectomies and are now well and one is awaiting resection of the anterior segment of the contralateral upper lobe.

\section{Discussion}

The increasing use of bronchography and tomography in the investigation of pulmonary tuberculosis has demonstrated abnormalities of the bronchi in association with tuberculous lesions in a high percentage of cases. That the bronchial abnormality has been due to tuberculous infection has been confirmed by the histological examination of our resected specimens. Clegg (1953) has recently drawn attention to the importance of ulcero-caseous tuberculous bronchitis as a method of spread in pulmonary tuberculosis. Many workers, of whom the most notable are Overholt, Woods, and Wilson (1953), have drawn attention to the segmental nature of the early tuberculous lesion. This confirms our experience and emphasizes the importance of the bronchus in the pathology of pulmonary tuberculosis. It is now widely accepted that resection is the treatment of choice for tuberculous bronchostenosis and tuberculous bronchiectasis. It seems logical, therefore, where these are associated with parenchymal disease that the same treatment where feasible should be used.

The aim of treatment in pulmonary tuberculosis should be sputum conversion while preserving as much pulmonary function as possible. Because of the frequency of bronchial involvement, sputum conversion is in our opinion best achieved by the total removal of the lesion together with its draining bronchus. Where this can be achieved safely we consider it to be the treatment of choice.

We believe the following factors are important in making resection a reliable and safe procedure.

In the pre-operative investigation, the extent and localization of the lesion are accurately determined, with particular reference to the bronchial element as shown by bronchography and tomography. A diligent search for the tubercle bacillus is carried out, and, wherever possible, sensitivity tests are performed. Bronchoscopy is performed if active tuberculous endobronchitis is suspected at the site of the section and respiratory function tests are performed in assessing the doubtful case.
Pre-operative treatment in the form of antibiotics and posture are of paramount importance, special emphasis being placed on the latter as a method not only of protecting the contralateral lung during the waiting period but also of reducing cavities and so lessening the risk of operative spread.

In the operative approach, such points as mobilizing the lung to the hilum, the method of bronchial closure, and the placing of drainage tubes for post-operative chest drainage are emphasized. Post-resection thoracoplasty is also considered to be of great importance.

Post-operative care is based on the principles of rapid and complete space obliteration and the early recognition and treatment of complications. To achieve these ends a properly equipped postoperative recovery ward is important, but teamwork on the part of experienced nursing staff, physiotherapists, and doctors is essential.

\section{Summary}

The results of 266 consecutive resections for pulmonary tuberculosis on 259 patients have been presented.

The indications, pre-operative investigation and treatment, operative and post-operative measures considered to be of importance have been described.

There has been an operative mortality of $1.88 \%$ and in $94.97 \%$ of patients the disease appears to be arrested.

Resection as a method of treatment and factors which make this a safe procedure have been discussed.

The surgery has been done by Mr. Dillwyn Thomas and the authors under his guidance. Our thanks are due to him for permission to publish these results.

We also wish to thank Dr. E. Williams for assistance in compiling this paper, the Tuberculosis Officers of South Wales for their help in following up the patients, Miss Verna Davies for the diagrams, and Mrs. B. Marshall and Mr. Gwilym Jones for the photographs.

\section{REFERENCES}

Bailey, C. P. (1947). J. thorac. Surg., 16, 328.

Bickford, B. J., Edwards, F. R., Esplen, J. R., Gifford, J. H., Mair, A. M., and Thomas, O. F. (1951). Thorax, 6, 25.

Clegg, J. W. (1953). Ibid., 8, 167.

Himmelstein, A., Berry, F. B., and Read, C. T. (1950). J. thorac. Surg., 20, 866.

Overholt, R. H., Langer, L., Szypulski, J. T., and Wilson, N. J. (1946). Ibid., 15, 384.

-Wilson, N. J., and Gehrig, L. J. (1952). Dis. Chest, 21, 32.

- Woods, F. M., and Wilson, N. J. (1953). Ibid., 23, 255.

Sellors, T. H., and Hickey, M. D. (1949). Thorax, 4, 82. 